\title{
Using word reading time in text processing research
}

\author{
TIMOTHY A. POST \\ University of Pittsburgh, Pittsburgh, Pennsylvania
}

\begin{abstract}
A study that employed a moving-window task was designed to test the paradigm's sensitivity to different levels of interclause integration. The program that controlled the task, implemented on a Terak 8510/a microcomputer in UCSD Pascal, is described. The results of the study indicate that word reading time is indicative of lower and "midlevel" types of integration, but not higher order domain knowledge considerations.
\end{abstract}

During the past decade, research on the processing of text has benefited from advances in experimental methods, particularly those employing computers. Paradigms have been devised that allow the measurement of fine-grained behaviors under subtle experimental manipulations. These developments have enhanced text processing research, particularly with respect to questions of encoding and representation. This paper describes a study that employs one such paradigm, the moving-window task (Just, Carpenter, \& Woolley, 1982), and discusses a few methodological implications of the findings for text processing research.

The program of research for the current effort is focused on understanding the role of domain knowledge during text processing. Earlier work performed in the laboratory of James Voss (Learning Research and Development Center, University of Pittsburgh) on this topic has suggested that the presence of knowledge of a domain by an individual results in comprehension that is quantitatively and qualitatively different from that by an unknowledgeable subject (Chiesi, Spilich, \& Voss, 1979; Spilich, Vesonder, Chiesi, \& Voss, 1979). In particular, high-knowledge individuals (HKs) recalled more text than low-knowledge subjects (LKs). Further, most of this advantage was found for the more important information, as defined by the goal structure of the domain. Later studies that used on-line verification paradigms (Post \& Voss, 1984) suggested that the above recall findings were due to differences in encoding. These experiments showed differences in the rate of verification accuracy and speed as a function of knowl-

Comments on an earlier draft of this paper by Doris Aaronson, David Dorfman, and James Voss added to its quality. The work described in this paper was performed under the direction of James Voss at the Learning Research and Development Center at the University of Pittsburgh. The Center is supported in part as a research and development center by funds from the National Institute of Education (NIE), U.S. Department of Education. The opinions expressed do not necessarily reflect the position or policy of the NIE, and no official endorsement should be inferred. The author's mailing address is: Learning Research and Development Center, University of Pittsburgh, 3939 O'Hara Street, Pittsburgh, PA 15213. edge differences, provided the information being verified was related to the knowledge domain. This was primarily true of information implied, but not explicitly mentioned, in the text materials.

Since the above studies lead to the conclusion that subjects encode information as a function of their topical knowledge, a natural step to take next was to examine encoding behavior for influences of knowledge differences. Indeed, some initial efforts in this direction examined sentence reading time (SRT) in this manner (Post \& Voss, 1984). A finding of this work was that HKs read important sentences more quickly than LKs, provided that the important sentence was "schemarelated," or a typical event for the domain. Other important, but atypical, events caused both groups to increase their reading times drastically. These results suggest that schematic information is largely available to HKs on the basis of a minimal amount of low-level information, and at speeded rates.

This research provides an additional benefit at a more global level. The examination of encoding processes from a text processing point of view allows a consideration of the research on reading comprehension (e.g., Aaronson \& Scarborough, 1977; Just \& Carpenter, 1980) within the current context. This facilitates efforts aimed at constructing a convergent and complete model of comprehension. For example, one of the findings in the reading literature relevant to the current effort is that subjects spend more time processing the end of text constituents (e.g., phrases, sentences) than other loci. This additional processing time is generally believed to be indicative of integrative and other higher order processes that are the focus of text processing research. This connection between the two bodies of research might lead to a complete model of comprehension, ranging from accessing lexicon(s) to the construction of text macrostructure(s).

\section{A TEXT PROCESSING STUDY OF ENCODING}

It thus was desirable to establish that the integrative phenomena outlined by the reading studies were capable 
of reflecting the types of higher processes studied in the text processing work. In particular, the "sentence wrap-up" phenomenon of Just and Carpenter (1980) was selected as a focus of the current experiment. The Just and Carpenter model specifies that the end of sentences is where most interclause integration occurs. If this is the case, then a careful manipulation of context, aimed at allowing different types of integrations and inferences at the end of targeted sentences, should cause different amounts of wrap-up time. Our earlier work on the influences of domain structure during text processing facilitates the creation of such contexts.

\section{Method}

Paradigm. The moving-window task (Just et al., 1982) was employed. This task was selected because it had been shown to produce data that closely resembled gazeduration times collected in eye-movement studies, without many of the latter method's difficulties. The task involved the presentation of a paragraph of text on a computer screen, with all words appearing in normal positions. At the outset of the task, however, each letter in every word was replaced with a dash. This reduced the information available on the screen to the position and length of each word. The subject's task was to repeatedly demand individual words (in the current effort, with keypresses). With each demand, one word was revealed to the subject; all previous words were reobliterated with dashes. The time between keypresses was thus indicative of the amount of time spent processing each word. Although certain perceptual behaviors (e.g., parafoveal processing, regressions) were specifically suppressed by this method, subjects adapted to the task and comprehended material at satisfactory levels.

Hardware and software considerations. The movingwindow task was implemented on a Terak 8510/a microcomputer (Post \& Fox, 1982). This system consisted of two single-density floppy-disk drives, a detached keyboard, a CRT, and an LSI-11 16-bit processor. UCSD Pascal was used as the operating system and programming environment. A millisecond clock was added for timing purposes, and software to provide a serial connection to a VAX-911 system was constructed. Other than these minor adaptations, the system was used as delivered by the vendor.

The program (MW) that accomplished the current task had to change the screen display (i.e., to move the "window" through the text) rapidly and without noticeable interruption. At the same time, the interresponse intervals had to be recorded as the time to read each word. Whereas many systems are capable of doing this at very fast rates, the Terak's processor was slow enough to cause concern. The use of UCSD's operating system (which employs a pseudocode concept) further slows down processing, thus necessitating a software solution. MW was therefore designed to use an array of records to describe each screen of text. Each record contained a word string, its length in characters, and whether the word ended a sentence (termed "eos"), ended a text
1. Initialize routine.

2. Repest for all sereens:

2.1. PARSESCREEN: Repeat for all text lines within s screen:

2.1.1. Repeat for each word within a line:

2.1.1.1. Store word.

2.1.1.2. Calculate or atore word length, bol, eol, eos

statuses.

2.2. DASHSCREEN: Construct dashed screen on transparent

CRT.

2.3. MOVIT: Repeat with each subject demand:

2.3.1. Output current stimulus atring.

2.3.2. Create next stimulus string:

2.3.2.1. If eol, then next stimulus string is next

word; otherwise, next stimulua atring is

dashea and next word.

2.3.3. Record timestamp at response.

2.4. Record data.

3. End routine.

Figure 1. MW algorithm.

line ("eol"), or began a line ("bol"). ${ }^{1}$ Having this information available while moving the text window kept the screen-update processing to an acceptable minimum.

A description of the MW's algorithm is provided in Figure 1. The program had three main sections. PARSEIT created the text representation by parsing the text into individual words and calculating the word-length, bol, eol, and eos information. This structure was then used by the DASHSCREEN and MOVIT procedures. DASHSCREEN created the initial screen of dashes with the screen "turned off" (a Terak capability). After turning the screen on, MOVIT would then, upon subject demand, write out the current stimulus string, which would both obliterate the previous word and reveal the current word. The next stimulus string would then be constructed, which was dependent upon whether the current word appeared at the end of a text line (extra processing was required for these words). Finally, MOVIT would record a timestamp of the current response as soon as it occurred. A timestamp, or current time on the millisecond clock, was recorded rather than calculating the interval during MOVIT in order to reduce the processing performed by this routine.

This procedure, simplified for purposes of communication, had other complexities; for example, the positions of each word had to be updated with each keypress. The program could also be made more efficient. One way would be to extract the PARSEIT routine and implement it as a preprocessor that would parse the experimental texts and store the parsed structures on disk. Since this would have complicated disk management and the editing of the experimental materials, it 
was not done in the current effort. A second optimization would be to have PARSEIT more thoroughly parse the text, for example, produce a structure that would contain the replacement strings, their screen location, and eol information. This would significantly reduce the time required by MOVIT to update the screen. Necessarily, the time to process the text would be increased, which would increase the time between screen presentations. (A copy of MW's source code can be obtained by writing the author.)

Experimental design. Returning to the theoretical motivations for the current effort, an experiment was designed to test the relevant hypotheses mentioned above. The knowledge domain employed was knowledge of the game of baseball. This was the domain used in the previous research (i.e., Chiesi et al., 1979; Post \& Voss, 1984; Spilich et al., 1979), and as such provided a degree of familiarity with the types of inferences made by subjects who possess different degrees of baseball knowledge.

In order to produce the conditions necessary for this study, four baseball actions were selected, with the stipulation that each could be described by a single statement that hinged upon a single word. This meant that the processing of each targeted action would necessarily be deferred until the processing of the action's critical word. For example, one of the actions was "The umpire called the pitch a strike." The critical word here is "strike," since the umpire in the action has two choices (ball and strike) up until the critical word is revealed.

Each of these actions was placed in four different contexts in order to vary the types of inferences and implications possible at the critical word. The contexts were determined by combining two different factors. First, the target action's importance was manipulated at two levels: more and less important. Retuming to the example described above (see Figure 2), the calling of the strike was less important when the context was a non-

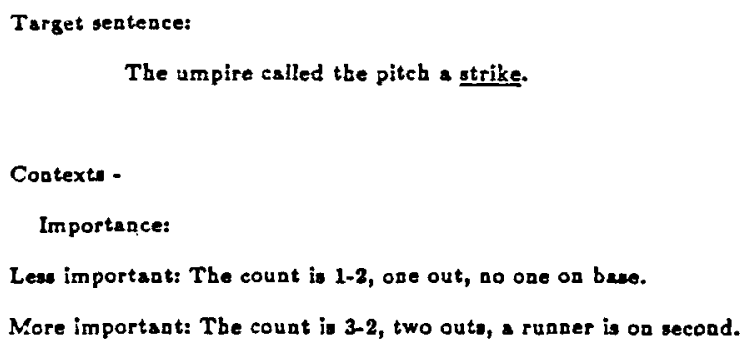

Constraint:

Constrained : The fitch is right over the plate.

Unconstrained : Here comes the pitch.

Alternate target sestence wording:

A strike is called by the umpire.

Figure 2. Manipulation example. critical pitch, that is, the count on the batter was zero or one strike. The more important context was produced by placing the action after the count on the batter had reached two strikes, with two outs, and runners in scoring position. This meant that the called strike would end the inning and eliminate the scoring opportunity, and enable summarizations of the batter's turn at bat, the scoring opportunity, and the half inning in progress. This is information that has been shown to be available to knowledgeable subjects immediately after processing similar target actions (Post \& Voss, 1984). The importance manipulation thus produces a large difference in the type of integrative processing possible at the critical word, and is particularly sensitive to the degree of baseball knowledge necessary for thorough comprehension.

The second context manipulation involved integrative processes at a more local level. A sentence that either constrained the target action to its actual meaning or was neutral and did not express any information with respect to the target action was placed before each target sentence. Referring to the example in Figure 2, the sentence "The pitch is right over the plate" implies that the pitch is a strike (in most situations). Having this information before the target action makes the latter's information redundant, and might allow or force any integration of it to text loci other than the targeted actions. The unconstrained sentence "Here comes the pitch" does not change the role of the critical word in the target action. This manipulation thus was designed to cause a shift of any integrative processing possible at the critical word to other loci. Further, the knowledge necessary to understand the relationship between the constrained sentence and the target sentence is minimal, and thus would not be expected to interact with this manipulation. Together with the importance manipulation, passages for the current experiment were constructed in order to test if the word reading time at the end of sentences was indicative of integrative processes at two levels of text abstraction. Each subject received each target action under each contextual manipulation.

Two between-subject factors were also employed. First, subjects were selected on the basis of performance on two tests: a baseball test that determined an individual's knowledge of the game of baseball, and a test of general reading comprehension ability (Davis \& Davis, 1957). The first test was used to select subjects who knew either much or little about the game of baseball. All of the subjects performed in a middle range on the latter test. The control of prior knowledge allowed the explicit testing of interactions between the degree of knowledge different subjects possessed and the above manipulations. Subjects were further divided into groups that received one of two possible wordings of the target sentence. It is conceivable that the end of a sentence has no special psychological status with respect to integrative processes. In other words, the extra time spent at constituent breaks might not indicate the integration of consituents with an individual's text representation. Thus, half of the subjects were exposed to target sentences 
that had the critical word appearing at the end of the sentence, whereas the others received the critical word earlier. If the end of a sentence does carry special status for interclause integration, then this comparison would reflect more processing time for the former subject group and differential processing as a function of the degree of integration possible. Eight subjects for each of the four groups were selected for this study.

Procedure. Subjects participated in this experiment in individual sessions. The instructions given to the subjects described the moving-window task and specified that they were to read each word as fully as possible, in order to obtain accurate estimates of the time needed to understand it within the context of the passage. Subjects were also aware that the domain in question was baseball, and that they would receive passages about four fictitious baseball games, with each passage containing four paragraphs. ${ }^{2}$ Advance notice of a summarization task, in which the subject would briefly summarize the important points of each game passage, was also given.

\section{Basic Findings}

Several interesting findings that are pertinent to the current discussion emerged in this experiment. First, the subjects who received the critical words at the end of the target sentences displayed longer word reading times (WRTs) than the other subjects. Further, this additional processing time was seen to be a function of the constraint manipulation; that is, processing time increased when the target sentence was unconstrained, for two of the four targets. This three-way interaction (critical word position, constraint, and particular target) was statistically significant $[F(3,84)=2.85, p<.05]$. The data for this interaction are displayed in Figure 3 in terms of the differences between the unconstrained and the constrained cells, for each of the targets and critical word position groups.

Examination of these results shows that the subjects who received the critical word early showed no influence of constraint or target. The other subjects did display a varying constraint effect. A posteriori analyses of the text materials lead to the conclusion that the degree of constraint effect was due to the typicality and/or the frequency of the target action. For example, the largest constraint effect was obtained with the "out" action, in which a base runner is thrown out stealing. Many of the high-knowledge subjects noted that this violated their expectations, as they expected that the runner would be safe, since runners usually are. On the other hand, the action displayed in Figure 2 (i.e., "strike") is a frequent and typical occurrence, and therefore may have reduced the formation of a specific expectation.

The other tests of effects for this analysis yielded highly consistent results, in that the target, position, and constraint factors, and all of their interactions, achieved significance in predictable directions. The importance and knowledge factors also yielded interesting results:

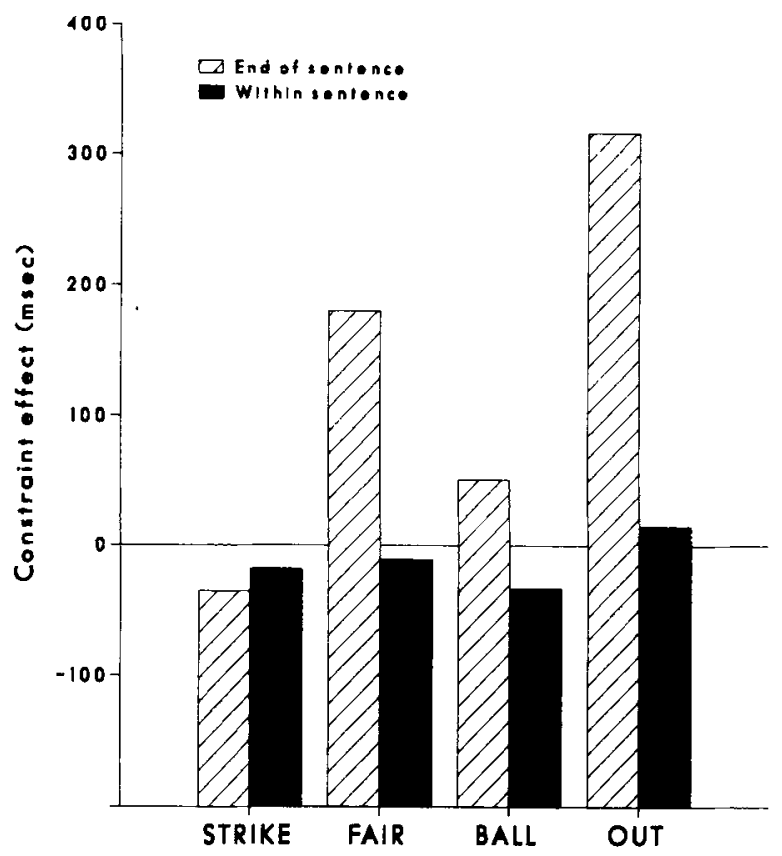

Figure 3. Constraint effects for each target and sentence position.

None of the 24 tests involving these two factors achieved statistical significance.

The lack of influence of the two knowledge-related factors was a consistent and interesting finding. At least four interpretations are possible. First, the subjects might not have been truly integrating the information. This is not likely, as summarizations that were collected for each passage indicated both better summarizations for HK subjects and an interaction with type of material (LK subjects recall mostly setting information). Further, the recall of the target actions showed that importance, knowledge, and particular target interacted (although this result is not as consistent as the WRT findings, and the knowledge $x$ importance interaction did not occur). These results, which replicate earlier recall findings (e.g., Spilich et al., 1979), suggest that the subjects did construct qualitatively and quantitatively different representations of the materials. The second interpretation is that knowledge-difference effects, and perhaps text-importance effects, are recall phenomena. This is tantamount to saying that a knowledgeable person should read a text at the same rate as a less knowledgeable individual. This was not quite the case in the current study-analysis of reading times for the noncritical words shows the HK subjects in general read the texts faster than the LKs. Furthermore, differences in sentence reading time have been found in other experiments for both knowledge (e.g., Post \& Voss, 1984) and importance (e.g., Cirilo \& Foss, 1980). The third interpretation is that reading occurs independently of knowledge-based comprehension. Again, the SRT effects just mentioned argue against this hypothesis. Also, in the current study some small (and albeit possibly spurious) 
knowledge effects were seen at the three words that followed the critical word for the subjects that received the passive target sentences.

The above line of reasoning leads me to believe that a partial autonomy exists between the lower "psycholinguistic" processes and those that involve text representation. Subjects may process "lower order" information for incorportation in knowledge structures with only slight increases in WRT. Because the end-of-sentence "wrap-up" effect (see Just \& Carpenter, 1980) is large, any knowledge effect may be overwhelmed by error variance, even under rigid experimental control. The general thrust of this explanation is that knowledge advantages during comprehension may not be a processing phenomenon, but rather may be due to abilities to instantiate long-term memory (LTM) structures (e.g., schemata, frames, scripts) very quickly, without requiring active resources. This leads to large differences in the text representations constructed by individuals with different levels of domain knowledge. The important methodological consideration is that the "implicit" capabilities of knowledgeable subjects mean that dif. ferent paradigms will access the advantages of knowledge to different degrees. Production tasks, such as recall, do provide for the more robust knowledge effects, since such activity relies primarily upon the aspects of comprehension that are most sensitive to knowledge.

\section{SOME IMPLICATIONS FOR ENCODING METHODOLOGY}

One important methodological issue raised by the above findings involves the difference in the SRT and WRT methods. After all, a SRT is composed of individual WRTs; one should be able to aggregate the WRTs and produce an equivalent SRT. Several such aggregate analyses were done for the current data, but none yielded new findings. The two paradigms, then, may yield different types of results. The difference is possibly due to task demands. In simple declarative sentences, as are most usually used in SRT studies, subjects demand a text segment once they've comprehended the sentence. That is, SRTs, because of the task's instructions, may reflect both the sum of the WRTs and the time to integrate the propositional information contained within the single sentence, which most usually is focused at a specific level (i.e., a particular sentence in a SRT experiment is homogeneous with respect to content). In the WRT paradigm, however, subjects demand a new stimulus when they've comprehended a word. Unless subjects process each word fully upon apprehension, which, in the strict sense, is a difficult discipline to adhere to, then integration effects will be spread across different words, and perhaps even sentences. As the level of integration increases, this spread will occur across larger text segments. Theoretical findings thus may be affected by this type of methodological consideration.
Data from the current study support the above observation in a couple of ways. For example, the overall mean WRT was roughly $500 \mathrm{msec}$, which is at least twice the usual amount of time spent fixating on words in eye-movement studies (Just \& Carpenter, 1980). This added processing time, which is principally due to the method employed, may have allowed much of the higher order integration to occur at many text loci other than intended. That the constraint effect yielded expected results is likely due to the different level of comprehension addressed by it. In particular, the constraining sentence for the example in Figure 2 precisely makes the critical word "target" redundant, and the critical WRT is thus able to reflect this consideration.

Since WRT may not reflect encoding influences of knowledge and domain structure, the issue of what paradigms can be employed remains to be addressed. As mentioned above, the sentence paradigm has been employed with some success in both our lab (Post \& Voss, 1984) and others (e.g., Bower, Black, \& Turner, 1979; Cirilo \& Foss, 1980). An intermediate solution that may help is the presentation of text constituents of different sizes (e.g., phrases). Indeed, other papers in this symposium directly address this issue. Also, a recent paper by Daneman and Carpenter (1983) has used a modified moving-window task, with some chunking of nonfunction words, in an investigation of inter- and intrasentential integration. It should be noted, however, that this level of comprehension is still lower than the order of macropropositions employed in the domainrelated studies.

This leads to a final caveat regarding the role of knowledge during encoding, and how research may help to outline and model this role. The possible extremes of this role are: Domain knowledge causes a comprehender to do more processing during encoding, and domain knowledge allows a comprehender to do less processing during encoding. Either position could be (and has been) taken in theories of comprehension. It is perhaps parsimonious to view the reduction in reading time as indicative of the latter view (i.e., having domain knowledge during processing allows one to "dredge up" prototypical or templated representations of actions in text). However, evidence is beginning to accumulate that suggests that, regardless of whether or not an individual employs explicit domain-related processing during reading, the locus of such activity is imprecise, and operates concurrently with the basic processes of reading. This suggests that a complete model of comprehension must integrate radically different empirical findings, that is, that no single paradigm will suffice. Results from production paradigms, such as recall, must be accounted for, providing that reconstruction be taken into account. Two other methodologies may also provide a clearer picture of the role of knowledge in encoding. Since encoding speed alone has limited application, dual tasks will test hypotheses related to effort. The work of Britton and Tesser (1982) and that of Daneman and Carpenter (1980) are examples of dual tasks. Another approach 
is to use the pupil-diameter data available from many of the eye-tracking machines. This measure has been related to level of arousal and cognitive effort (Kahneman, 1973), and might be studied along with eye-fixation data. This type of data collection is rigorous, expensive, and difficult; other methods should be explored first.

\section{REFERENCES}

Aaronson, D., \& Scarborough, H. S. (1977). Performance theories for sentence encoding: Some quantitative models. Jour. nal of Verbal Learning and Verbal Behavior, 16, 277-303.

Bower, G. H., Black, J. B., \& Turner, T. J. (1979). Scripts in memory for text. Cognitive Psychology, 11, 177-220.

Britton, B. K., \& Trs8ER, A. (1982). Effects of prior knowledge on use of cognitive capacity in three complex cognitive tasks. Journal of Verbal Learning and Verbal Behavior, 21, 421-436.

Chiesi, H. L., Spilich, G. J., \& Voss, J. F. (1979). Acquisition of domain-related information in relation to high and low domain knowledge. Journal of Verbal Learning and Verbal Behav. ior, 18, 257-274.

CiriLo, R. K., \& Foss, D. J. (1980). Text structure and reading time for sentences. Journal of Verbal Learning and Verbal Behavior, 19, 96-109.

Daneman, M., \& Carpenter, P. A. (1980). Individual differences in working memory and reading. Journal of Verbal Learning and Verbal Behavior, 19, 450-466.

Daneman, M., \& Carpenter, P. A. (1983). Individual differences in integrating information between and within sentences. Journal of Experimental Psychology: Learning, Memory, and Cognition, 9, 561-584.

Davis, F. B., \& Davis, C. C. (1957). Davis Reading Test, Form 1A, Series 1. New York: The Psychological Corporation.
Just, M. A., \& Carpenter, P. A. (1980). A theory of reading: From eye fixations to comprehension. Psychological Review, 87, 329-354.

Jugt, M. A., Carpenter, P. A., \& Woolley, J. D. (1982). Paradigms and processes in reading comprehension. Journal of Experimental Psychology: General, 111, 228-238.

Kahneman, D. (1973). Attention and effort. Englewood Cliffs, NJ: Prentice-Hall.

Post, T. A., \& Fox, J. L. (1982). Programming experiments on a TERAK 8510/a microcomputer. Behavior Research Methods \& Instrumentation, 14, 276-280.

Post, T. A., \& Voss, J. F. (1984). On-line effects during the processing of domain-related text by high-and low-knowledge individuals. Manuscript in preparation.

Spilich, G. J., Vesonder, G. T., Chiesi, H. L., \& Voss, J. F. (1979). Text processing of domain-related information for individuals with high and low domain knowledge. Journal of Verbal Learning and Verbal Behavior, 18, 275-290.

\section{NOTES}

1. An array was used instead of a more elegant strategy (e.g., pointers and dynamic storage) because of the limits of memory available. It was decided that it would be better to know how much memory for text storage was available before running the program, rather than during execution.

2. The actual design, which employed four Graeco-Latin squares, was intended to balance different levels of order effects. Thus, each fictitious game was written so that each target word would appear in one of the four contexts, and each of the four contexts would occur for each subject. Across the four passages, then, each subject received each target under each condition, and both within- and between-subjects order effects were balanced. 